\title{
MicroRNA-593-3p regulates insulin-promoted glucose consumption by targeting Slc38a1 and CLIP3
}

\author{
Xilin Yang', Zezhang Tao', Zhanyong Zhu², Hua Liao', Yueqiang Zhao² and \\ Huajun Fan² \\ 1Department of Otorhinolaryngology - Head and Neck Surgery, Renmin Hospital of Wuhan University, \\ Wuhan, China \\ 2Department of Plastic Surgery, Renmin Hospital of Wuhan University, Wuhan, China
}

Correspondence should be addressed to Z Tao or Z Zhu

Email

taozezhang@hotmail.com or zyzhu@whu.edu.cn

\begin{abstract}
Insulin plays an important role in the regulation of glucose metabolism. However, the molecular mechanisms involved are not entirely clarified. In this context, we found that miR-593-3p negatively regulates insulin-regulated glucose metabolism in hepatocellular carcinoma HepG2 and Bel7402 cells. We then identified SIc38a1 and CLIP3 as novel targets of miR-593-3p. Further studies demonstrated that SIc38a1 and CLIP3 mediate insulin-regulated glucose metabolism. Interestingly, we also demonstrated that miR-593-3p expression was negatively associated with SIc38a1 and CLIP3 expression in insulin-treated HepG2 cells, and insulin-induced SIc38a1 and CLIP3 expression via downregulation of miR-593-3p. Taken together, this study indicates that inhibition of miRNA-593-3p by insulin promotes glucose metabolism through the regulation of Slc38a1 and CLIP3 expression, and provides a new insight into the role and mechanism of insulin-induced glycolysis.
\end{abstract}

\author{
Key Words \\ - miR-593-3p \\ - Slc38a1 \\ - CLIP3 \\ - insulin
}

\section{Introduction}

Insulin is an important peptide hormone produced by pancreatic $\beta$-cells (Moore \& Cooper 1991, Sonoda et al. 2008). It promotes the uptake of glucose from the blood to skeletal muscles and fat tissue; therefore, it plays a central role in the metabolism of fats and carbohydrates (Thirone et al. 2006, Liao et al. 2010). When control of insulin levels fails, glucose metabolism disorders can result and can culminate in diabetes. Disturbances of glucose metabolism are also present in cancer (Tavana \& Zhu 2011, Ying et al. 2012).

MicroRNAs (miRNAs) are 19-23-nucleotide (nt) non-coding RNAs processed from double-stranded hairpin precursors that function as specific regulators of gene expression by targeting sequences with partial complementarity in the 3 '-untranslated regions (3'-UTRs) of mRNAs, resulting in mRNA degradation (Lai 2002, Krol et al. 2010). A number of miRNAs have been shown to regulate insulin secretion. For example, miR-184 inhibits insulin secretion through the repression of its target Slc25a22 (Morita et al. 2013). miR-375 also negatively regulates insulin gene expression in INS-1E cells by directly targeting PDK1 (El Ouaamari et al. 2008). Overexpression of miR-34a in MIN6 cells leads to reduced insulin secretion through its target protein VAMP2 
(Lovis et al. 2008). Contrastingly, relatively few studies have examined the role of miRNAs in insulin-regulated glucose metabolism.

Solute carrier family 38 member 1 (Slc38a1, type A amino acid transporter 1), also referred to as SNAT1 and ATA1, is a glutamine transporter (Sugawara et al. 2000, Xie et al. 2014). Slc38a1 is responsible for system A transport activity (Sugawara et al. 2000, Xie et al. 2014) and has been widely reported to take part in a variety of physiological cellular processes, including nutrient uptake, metabolic regulation and malignant transformation (McGivan \& Pastor-Anglada 1994, Ogura et al. 2012). CAP-Gly domain containing linker protein 3 (CLIP3) is a cytoplasmic protein that is targeted to the plasma membrane and trans-Golgi network membranes (Deng et al. 2012). Some reports have found that CLIP3 is involved in the regulation of membrane trafficking and early Golgi network dynamics (Lallemand-Breitenbach et al. 2004, Sorice et al. 2010). However, the roles of Slc38a1 and CLIP3 in insulin-stimulated glucose metabolism have not been demonstrated.

Presently, two studies have reported the regulation of glucose metabolism by insulin, and explored the underlying mechanisms (Li et al. 2013, 2014). These studies showed that insulin promotes glucose uptake and glycolysis in hepatocellular carcinoma cells by miR-99a/ mTOR/PKM2 and ROS/PKM2 pathways (Li et al. 2013, 2014). Based on these previous findings, a search for potential miRNAs that could play a role in insulin-regulated glucose metabolism using miRNAs was conducted. This led to the identification of miR-593-3p, which this study confirmed plays a role in insulin-regulated glucose energy metabolism. miR-593-3p potentially targets Slc38a1 and CLIP3, which also participates in insulin-regulated glucose energy metabolism. This study provides new insights into miRNA expression and insulin-regulated signaling pathways.

\section{Materials and methods}

\section{Cell lines, culture and transfection}

Mouse myoblasts cell line $\mathrm{C} 2 \mathrm{C} 12$ and human hepatocellular carcinoma cell lines HepG2 and BeL7402 were obtained from China Center for Type Culture Collection. Cells were cultured in Dulbecco's modified Eagle's medium supplemented with 10\% heat-inactivated fetal bovine serum, $100 \mu \mathrm{g} / \mathrm{mL}$ penicillin, and $100 \mu \mathrm{g} / \mathrm{mL}$ streptomycin, and cells were maintained in humidified $5 \% \mathrm{CO}_{2}$ incubator at $37^{\circ} \mathrm{C}$. The transfection was carried out using Lipofectamine 2000 (Invitrogen) in accordance with the manufacturer's instructions.

\section{Reagents}

Insulin and antibody against Slc38a1 and CLIP3 were purchased from Sigma. Antibody against $\beta$-tubulin was purchased from Santa Cruz Biotechnology. Chemically synthesized miRNA mimics and miRNA inhibitors were purchased from RiboBio (Guangzhou, China). The short hairpin RNA (shRNA) and irrelevant control shRNAs were purchased from GenePharma (Shanghai, China) and target sequences are listed in Supplementary Table 1 (see section on supplementary data given at the end of this article).

\section{Ethics statement}

The study was conducted according to the principles of the Declaration of Helsinki and approved by the Institutional Review Board of Renmin Hospital of Wuhan University in accordance with its guidelines for the protection of human subjects. All participants provided written informed consent to participate in the study.

\section{Clinical specimens}

A total of 41 liver cancer specimens and 41 matched adjacent non-tumorous tissues used to analyze miR-593-3p, Slc38a1 and CLIP3 mRNA levels were collected from the surgical specimen archives of the Renmin Hospital of Wuhan University, from February 2010 to February 2016. Tissue samples were cut into two parts: one was reviewed by two expert pathologists to verify the histologic diagnosis, the other immediately snap-frozen in liquid nitrogen and stored in liquid nitrogen until RNA extraction was performed. Tumors were staged according to the American Joint Committee on Cancer pathologic tumor-node metastasis classification. The characteristics of patients are described in Supplementary Table 2.

\section{Primary human hepatocyte cultures}

Thirty-six healthy liver tissue samples were obtained from the unused portions of donor livers used for liver transplantation. Hepatocytes with viability over 90\% were seeded at $1 \times 10^{6}$ cells/well in 12 -well biocoat plates in DMEM supplemented with 5\% FBS, $100 \mathrm{U} / \mathrm{mL}$ penicillin, $100 \mu \mathrm{g} / \mathrm{mL}$ streptomycin, $4 \mu \mathrm{g} / \mathrm{mL}$ insulin and $1 \mu \mathrm{M}$ dexamethasone. After attachment at $37^{\circ} \mathrm{C}$ in a humidified

Published by Bioscientifica Ltd 
atmosphere of $5 \% \mathrm{CO}_{2}$, hepatocytes were cultured in complete William's Medium E (WME) and overlaid with Matrigel $(0.25 \mathrm{mg} / \mathrm{mL})$. Cell culture medium was replaced on a daily basis.

\section{Measurement of glucose consumption and lactate production}

Measurement of glucose consumption and lactate production was performed as described in Li et al. (2013, 2014). In brief, cells were treated as indicated and the supernatants of cell culture medium were collected. The media were assayed immediately by using glucose assay kit and lactate assay kit (BioVision) according to the manufacturer's protocol. The glucose consumption and lactate production were normalized to cell number.

\section{Western blot}

Proteins were separated on a 7.5 or $10 \%$ sodium dodecyl sulfate-polyacrylamide gel and transferred on to a nitrocellulose membrane (Bio-Rad). Then, the membrane was blocked with 5\% non-fat milk and incubated with primary antibodies. Horseradish peroxidase-linked antirabbit, anti-mouse or anti-goat antibodies (Santa Cruz Biotechnology) were used as secondary antibodies. Blots were developed using SuperSignal Chemiluminescent reagent (Pierce) and the stained membranes were analyzed with a LAS-4000 image document instrument (FujiFilm, Tokyo, Japan).

\section{RNA extraction and real-time PCR}

Total cellular RNA was extracted from cells using TRIzol reagent (Invitrogen). miRNA reverse transcription reactions were carried out using the miRNA reverse transcription kit (Life Technologies). Real-time PCR for miRNA expression levels was performed using miRNAspecific TaqMan MGB probe and TaqMan universal master mix in an Applied Biosystem 7900HT PCR system. miRNA expression levels were normalized to the internal control small RNAs: RNU6 and RNU48. MRNA samples were reversed transcribed into cDNA using a reverse transcription kit (Invitrogen). Real-time PCR for mRNA expression levels was performed using the Roche LC480 and SYBR RT-PCR kits (DBI Bioscience, Ludwigshafen, Rhineland-Palatinate, Germany). MRNA expression levels were normalized to the GAPDH. Results expressed as $2^{\text {-dCt }}$ (delta cycle threshold) calculated using the above-mentioned housekeeping small RNAs or GAPDH. Primers used in this study are listed in Supplementary Table 3.

\section{miRNA microarray}

Microarray assay was done by KangChen Inc (Shanghai, China). In brief, HepG2 cells were starved in serum-free medium for $24 \mathrm{~h}$. Then, the cells were exposed to $200 \mathrm{nM}$ insulin for $6 \mathrm{~h}$. Total RNA was isolated using MirVana miRNA isolation kit (Applied Biosystems), according to the manufacturer's instructions. RNA from each sample was quantified using the NanoDrop ND-1000. For miRNA microarray analysis, Agilent Array platform was used. The sample preparation and microarray hybridization were performed based on the manufacturer's standard protocols. Data analysis was completed by KangChen and taking a threefold difference as the cut-off point.

\section{Luciferase assays}

After transfection, cells were lysed with the Luciferase Cell Culture Lysis reagent (Promega). Then, the cell lysates and luciferase assay substrate (Promega) were mixed before the light intensity was detected using a luminometer (GloMax 20/20, Promega). All reporter assays were repeated for at least three times. Results are expressed as the mean value relative to the vector control, which was arbitrarily assigned as 1 .

\section{Statistical analysis}

Data analysis was performed using GraphPad Prism 6.0. (GraphPad Software) software. Unless stated otherwise, the Student's $t$-test or one-way analysis of variance followed by Dunnett's multiple comparisons test was performed to compare the differences between two groups or more than two groups, respectively. Correlations were assessed using Pearson's $R$. A $P$ value of less than 0.05 was considered statistically significant.

\section{Results}

Insulin promotes glucose consumption and lactate production through miR-593-3p in hepatocellular carcinoma cells

To explore the role of miRNAs in insulin-regulated signaling, microarray analysis was performed to identify
○ 2016 Society for Endocrinology Printed in Great Britain
Published by Bioscientifica Ltd 
A



Insulin

C

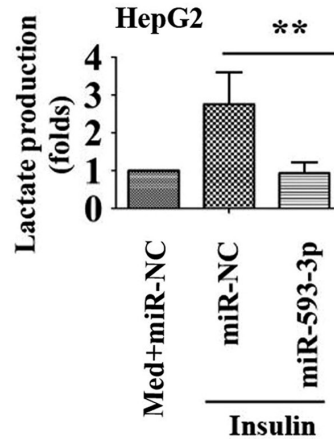

D

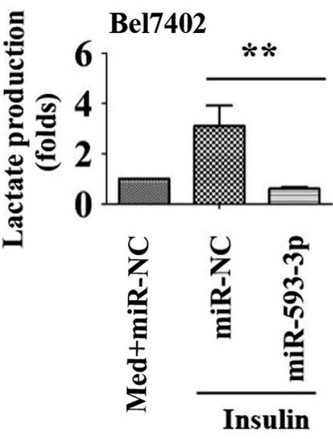

B
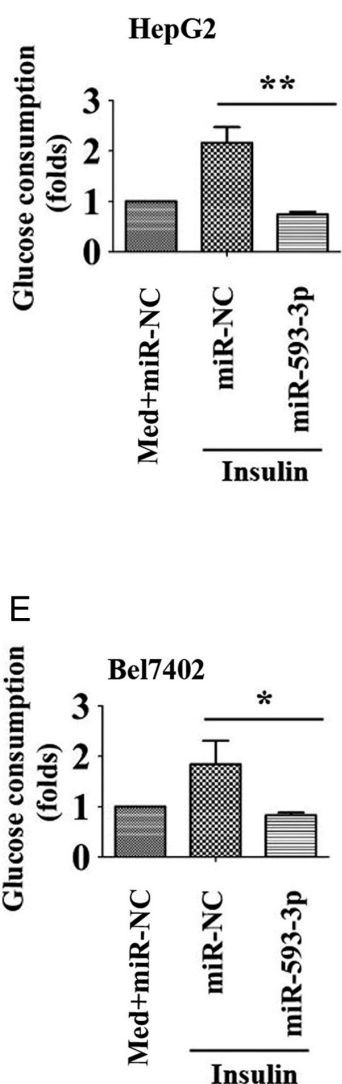

Figure 1

Involvement of miR-593-3p in insulin-stimulated glucose consumption and lactate production. (A) HepG2 cells were transfected with the non-specific miRNA inhibitor control (anti-NC) or indicated miRNA inhibitor. After transfection for $24 \mathrm{~h}$, cells were cultured in serum-free medium for $24 \mathrm{~h}$ and treated with or without insulin $(200 \mathrm{nM})$ for $12 \mathrm{~h}$ before analysis of the glucose consumption levels. (B and C) HepG2 cells were transfected with miRNA mimics control (miR-NC) or miR-593-3p mimics (miR-593-3p).

After transfection for $24 \mathrm{~h}$, cells were cultured in serum-free medium for $24 \mathrm{~h}$ and treated without or with insulin ( $200 \mathrm{nM})$ for $12 \mathrm{~h}$ before glucose consumption analysis (B) and lactate production levels (C). ( $D$ and $E$ ) Experiments were performed as in (B) and (C), except the Bel7402 cells were used. Bar graphs represent mean \pm s.D., $n=3$ $(* * P<0.01 ; * P<0.05)$. Med, medium. differentially expressed miRNAs in insulin-treated and -untreated HepG2 cells. This led to the identification of nine upregulated and three downregulated miRNAs in insulin-treated HepG2 cells compared with control HepG2 cells (Supplementary Table 4). Next, the effect of these altered miRNAs on insulin-regulated glucose energy metabolism was examined. Shown in Fig. 1A, among the 12 miRNAs, miRNA-148a-3p and miR-593-3p inhibitor oligonucleotides were able to regulate insulin-stimulated glucose consumption. miR-593-3p was chosen for subsequent studies that are reported here. Another ongoing project in this lab is investigating the relationship between miRNA-148a-3p and insulin-regulated glucose metabolism.

To confirm the miR-593-3p inhibitor results, the effect of expression of miR-593-3p on insulin-regulated glucose energy metabolism was studied. Consistent with the above results, miR-593-3p mimics reduced insulinstimulated glucose consumption and lactate production in HepG2 cells (Fig. 1B and C). This effect was not celltype specific because overexpression of miR-593-3p also inhibited insulin-stimulated glucose consumption and lactate production in Bel7402 cells (Fig. 1D and E).
If this miR-593-3p is not well expressed, many of the observations may simply be artifacts. Therefore, we explored the expression of miR-593-3p. In real-time PCR analysis, miR-593-3p levels in primary human hepatocytes and HepG2 cells were approximately threefold less than miR-122. This has been reported as high expression in the liver (Hsu et al. 2016) (Supplementary Fig. 1A and B).

The efficiency of either overexpression or suppression of miR-593-3p by oligonucleotide mimics or inhibitors was also explored. Real-time PCR results showed that transfection with miR-593-3p mimics resulted in a remarkable increase of miR-593-3p levels in HepG2 cells. Whereas the miR-593-3p inhibitor could lead to a reduction of miR-593-3p levels compared with controls (Supplementary Fig. 1C and D). In addition, the effect of miR-593-3p and anti-miR-593-3p on glucose consumption and lactate production in the absence of insulin was also analyzed. Supplementary Fig. 2A and B shows miR-5933 p-inhibited glucose consumption and lactate production in a dose-dependent manner. Collectively, the data indicated that miR-593-3p could inhibit insulin-regulated glucose energy metabolism. 


\section{SIc38a1 and CLIP3 are potential miR-593-3p target genes involved in insulin-stimulated glucose consumption and lactate production}

Two programs for miRNA target prediction (TargetScan Human and miRDB) were used to predict miR-593-3p targets. Several high-scoring candidate targets (Slc38a1, CLIP3, Stac2, BAP1 and TRIM3) and three reported targets (PROP1, MFF and PLK1) were selected, and then their coding sequences were cloned into plasmid expression vectors. The rest predicted target genes are listed in Supplementary Table 5. The effect of each of these genes on insulin-stimulated glucose consumption was examined. The results showed that only Slc38a1 and CLIP3 overexpression regulated insulin-stimulated glucose consumption (Fig. 2A). To further determine the role of Slc38a1 and CLIP3 in insulin-stimulated glucose consumption and lactate production, we synthesized specific shRNAs for Slc38a1 and CLIP3 and tested their efficiency (Fig. 2B). shRNA-Slc38a1\#2 and shRNACLIP3\#1 were selected for the experiments described below. Insulin-stimulated glucose consumption and lactate production was suppressed by shRNAs for Slc38a1 and CLIP3 (Fig. 2C and D). Similar results were also obtained in Bel7402 cells (Fig. 2E and F). Furthermore, the effect of Slc38a1 and CLIP3 on glucose consumption and lactate production in the absence of insulin was also estimated. Shown in Supplementary Fig. 2C-F, Slc38a1and CLIP3-induced glucose consumption and lactate production in a dose-dependent manner. These results show insulin-stimulated glucose consumption and lactate production through Slc38a1 and CLIP3.

\section{SIc38a1 and CLIP3 is a direct target gene of miR-593-3p}

As miR-593-3p, Slc38a1 and CLIP3 were involved in insulin-stimulated glucose consumption and lactate production, it needs to be determined whether or not miR-593-3p regulates Slc38a1 and CLIP3 expression by posttranscriptional targeting of their $3^{\prime}$ UTRs. The wildtype Slc38a1 3' UTR and a mutated version with ablation of the miR-593-3p predicted target sites were cloned into a firefly luciferase reporter plasmid (Fig. 3A). Reporter assays indicated that the miR-593-3p mimic effectively reduced luciferase activity controlled by the wild-type Slc38a1 3'-UTR. However, the miR-593-3p mimic did not affect luciferase activity of the mutated Slc38a1 3'-UTR (Fig. 3B).
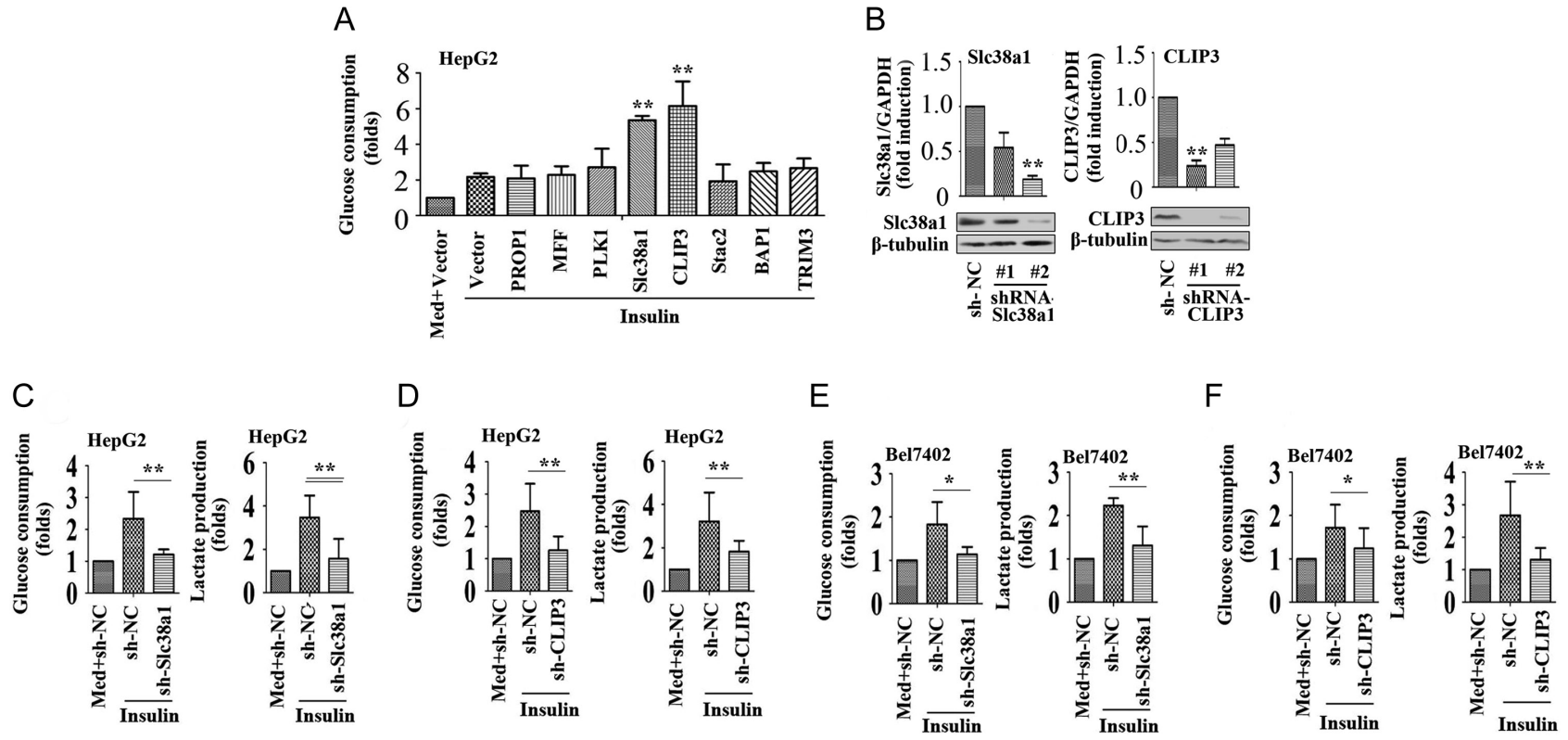

Figure 2

Involvement of SIc38a1 and CLIP3 in insulin-stimulated glucose consumption and lactate production. (A) HepG2 cells were transfected with the control vector or expression vectors for indicated genes. After transfection for $24 \mathrm{~h}$, cells were cultured in serum-free medium for $24 \mathrm{~h}$ and treated without or with insulin $(200 \mathrm{nM})$ for $12 \mathrm{~h}$ before analysis of the glucose consumption levels. (B) HepG2 cells were transfected shRNA-control or shRNA-SIc38a1 (left panel) or shRNA-CLIP3 (right panel) for $48 \mathrm{~h}$ before real-time PCR and Western blot analyses. (C, D) HepG2 cells were transfected with shRNAcontrol, shRNA-SIc38a1 (C) or shRNA-CLIP3 (D). After transfection for $24 \mathrm{~h}$, cells were cultured in serum-free medium for $24 \mathrm{~h}$ and treated with or without insulin $(200 \mathrm{nM})$ for $12 \mathrm{~h}$ before analysis of glucose consumption and lactate production levels. (E and F) Experiments were performed as in (E) and (F), except the Bel7402 cells were used. Bar graphs represent mean \pm S.D., $n=3(* * P<0.01 ; * P<0.05)$. Med, medium.

http://jme.endocrinology-journals.org DOI: 10.1530/JME-16-0090
๑) 2016 Society for Endocrinology Printed in Great Britain
Published by Bioscientifica Ltd 
A

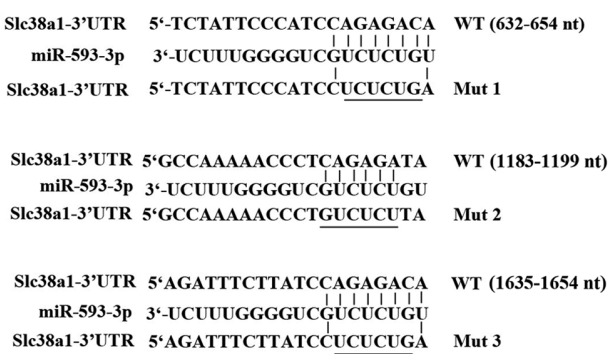

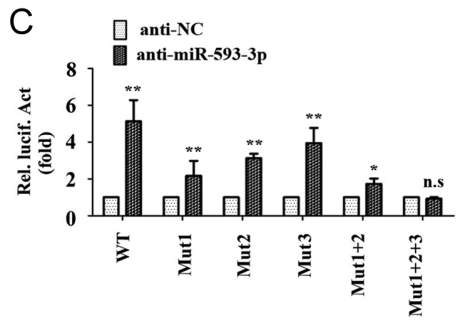

Slc38a1-3'UTR

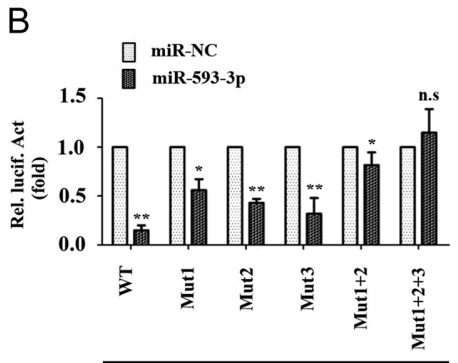

SIc38a1-3'UTR
D

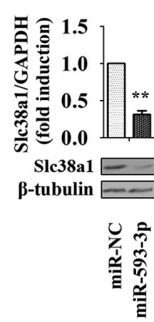

E

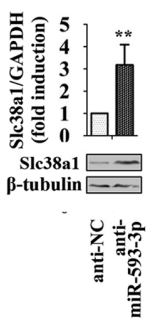

Figure 3

Analysis of SIc38a1 3'-UTR and targeting of miR-593-3p. (A) The predicted miR-593-3p targeting site in the SIc38a1 3 '-UTR and the UTR mutant. (B and C) HepG2 cells were transfected with WT or indicated mutants of the SIc38a1 3'- UTR reporter plasmids and miR-593-3p (B) or anti-miR-593-3p (C). After $48 \mathrm{~h}$, the luciferase activities were analyzed. (D) HepG2 cells were transfected with miRNA mimics control (miR-NC) or miR-593-3p mimics for $48 \mathrm{~h}$ before real-time PCR (upper panel) and Western blot (lower panel) analyses. (E) Experiments were performed as in (D), except non-specific miRNA inhibitor control or anti-miR-593-3p were used. Bar graphs represent mean \pm S.D., $n=3(* * P<0.01 ; * P<0.05)$.
Conversely, the miR-593-3p inhibitor increased luciferase activity controlled by the Slc38a1 3'-UTR (Fig. 3C), and this effect was not seen in the mutated $3^{\prime}$-UTR construct (Fig. 3C). The effect of miR-593-3p on endogenous Slc38a1 expression was also examined. Real-time PCR and Western blot results showed that the overexpression of miR-593-3p significantly decreased Slc38a1 mRNA levels (Fig. 3D). Contrarily, the miR-593-3p inhibitor resulted in induction of Slc38a1 mRNA expression (Fig. 3D).

Likewise, whether or not miR-593-3p can target the 3'-UTR of CLIP3 mRNA was investigated. Potential target sites of miR-593-3p and corresponding mutated sites in the 3 '-UTR of CLIP3 sequences are shown in Fig. 4A.
Overexpression of miR-593-3p decreased the luciferase activity of the wild-type reporter, and conversely antimiR-593-3p increased the luciferase activity as shown in Fig. 4B and C. However, when using the mutant CLIP3 3 '-UTR luciferase reporter plasmids, the luciferase activity was not significantly changed by either overexpression or inhibition of miR-593-3p (Fig. 4B and C). Similarly, the endogenous CLIP3 mRNA and protein level were efficiently reduced by miR-593-3p. While higher levels of CLIP3 mRNA and protein were observed in miR-593-3p inhibitor transfected cells (Fig. 4D). This data indicate that miR-593-3p targets the 3'-UTRs of Slc38a1 and CLIP3 and alters their expression.
A
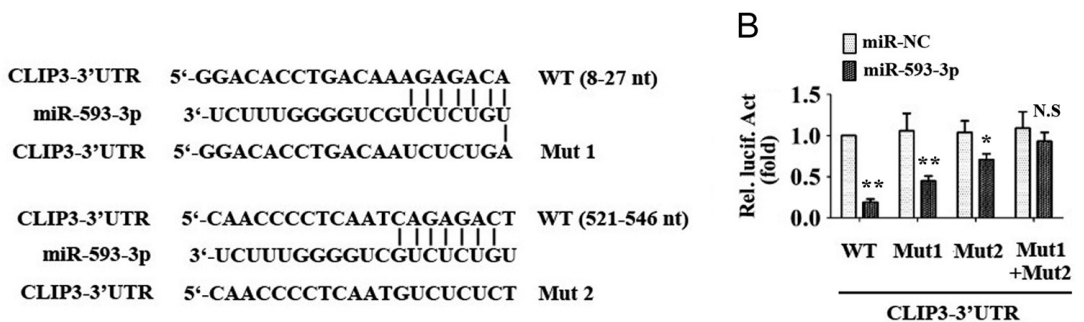

C

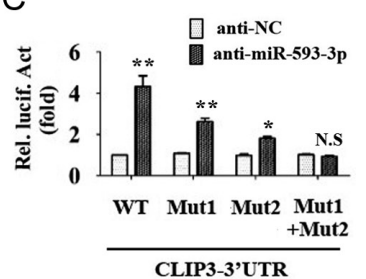

D

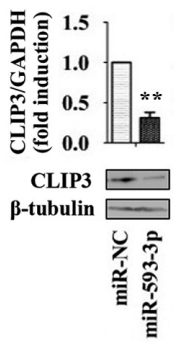

E

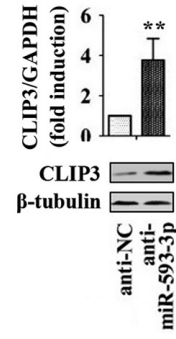

Figure 4

Analysis of CLIP3 3' UTR and targeting of miR-593-3p. (A) The predicted miR-593-3p targeting site in the CLIP3 3 '-UTR and the UTR mutant. (B and C) HepG2 cells were transfected with WT or indicated mutants of CLIP3 3'-UTR reporter plasmids and miR-593-3p (B) or anti-miR-593-3p (C). After $48 \mathrm{~h}$, the luciferase activities were analyzed. (D) HepG2 cells were transfected with miRNA mimics control (miR-NC) or miR-593-3p mimics for $48 \mathrm{~h}$ before real-time PCR (upper panel) and Western blot (lower panel) analyses. (E) Experiments were performed as in (D), except non-specific miRNA inhibitor control or anti-miR-593-3p were used. Bar graphs represent mean $\pm \mathrm{SD}, n=3\left({ }^{* *} P<0.01 ;{ }^{*} P<0.05\right)$. 
A

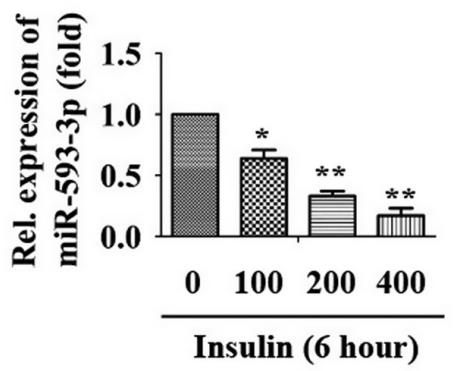

B

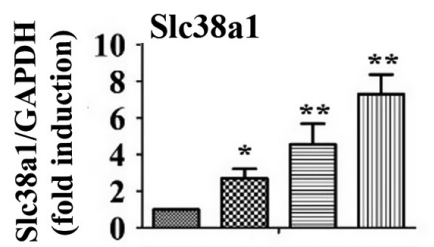

Slc38a1 ---

B-tubulin - - -

$\begin{array}{lllll}0 & 100 & 200 & 400\end{array}$

Insulin (6 hour)

C

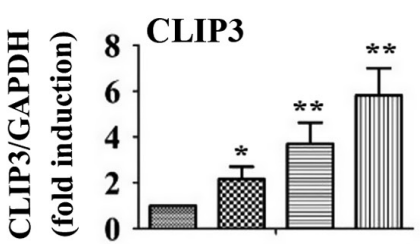

CLIP3

$\beta$-tubulin

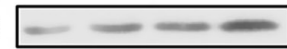

$\begin{array}{llll}0 & 100 & 200 & 400\end{array}$

Insulin (6 hour)

D



Slc38a1

B-tubulin
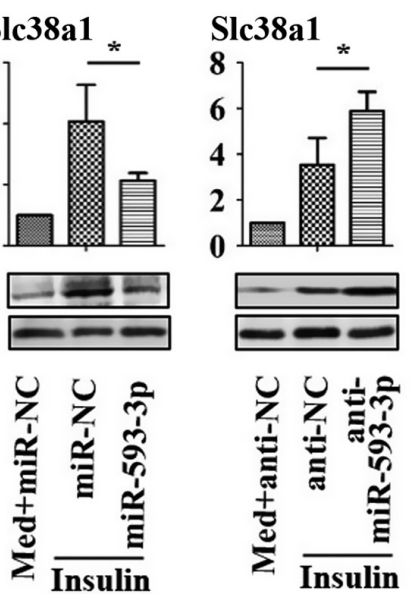
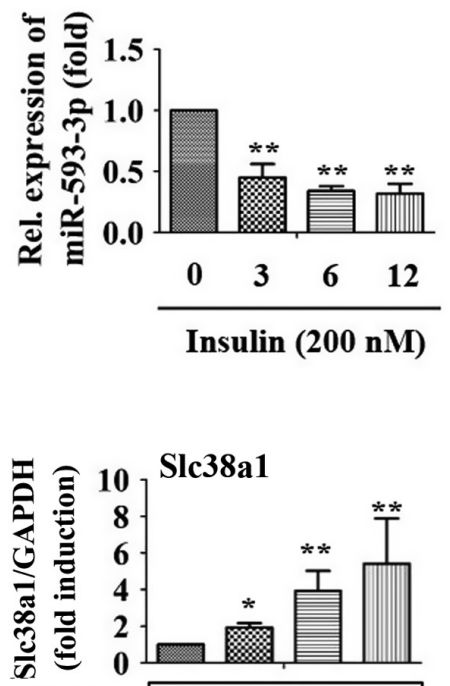

Slc38a1

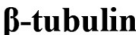

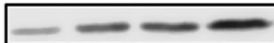

$-\cdots$

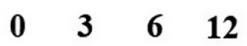

Insulin (200 nM)

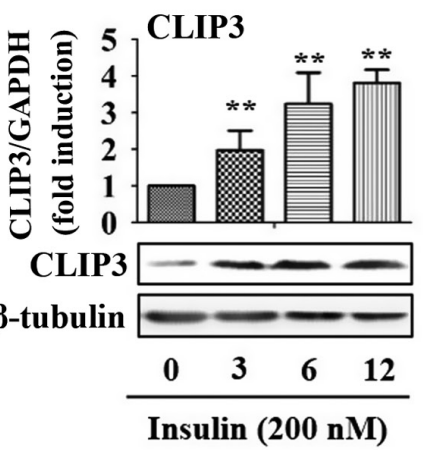

E

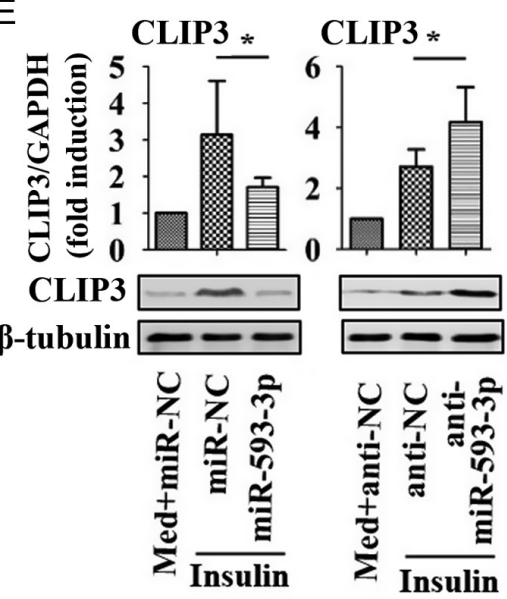

Figure 5

Insulin induces SIC38a1 and CLIP3 expression by inhibition of miR-593-3p. (A) HepG2 cells were starved in serum-free medium for $24 \mathrm{~h}$. Then, the cells were exposed to $0,100,200$ and $400 \mathrm{nM}$ of insulin for $6 \mathrm{~h}$ (left panel) or exposed to $200 \mathrm{nM}$ insulin for 0, 3, 6 and $12 \mathrm{~h}$ (right panel). miR-593-3p levels were determined by real-time PCR using $\mathrm{U} 6$ as an internal control. (B and $C$ ) Experiments were performed as in (A), except relative SIc38a1 (B) and CLIP3 (C) mRNA and protein levels were determined by real-time PCR (upper panel) and Western blot (lower panel) analyses. (D) HepG2 cells were transfected with miR-NC and miR-593-3p mimics (left panel) or anti-NC and miR-593-3p inhibitor (right panel). After transfection for $24 \mathrm{~h}$, cells were cultured in serum-free medium for $24 \mathrm{~h}$ and treated with or without insulin $(200 \mathrm{nM})$ for $6 \mathrm{~h}$. Relative Slc38a mRNA and protein levels were determined by real-time PCR and Western blot (lower panel) analyses. (E) Experiments were performed as in (D), except relative CLIP3 mRNA and protein levels were determined by real-time PCR and Western blot (lower panel) analyses. Bar graphs represent mean \pm s.D., $n=3(* * P<0.01 ; * P<0.05)$. Med, medium. 
The relationship between SIc38a1 and CLIP3 in insulin-regulated glucose metabolism

As Slc38a1 and CLIP3 are direct target genes of miR-593-3p, the relationship between Slc38a1 and CLIP3 in insulin-regulated glucose metabolism was tested. In real-time PCR assays, neither overexpression nor knockdown of Slc38a1 affected CLIP3 expression (Supplementary Fig. 3A). Likewise, CLIP3 did not affect Slc38a1 expression (Supplementary Fig. 3B). Whether or not Slc38a1 influences the effect of CLIP3 on insulin-regulated glucose metabolism was tested next. Shown in Supplementary Fig. 3C, overexpression of Slc38a1 abolished the effect of sh-CLIP3 on insulinregulated glucose metabolism. Similar results were also obtained in CLIP3 overexpressing HepG2 cells (Supplementary Fig. 3D). Co-knockdown of Slc38a1 and CLIP3 synergistically decreased insulin-regulated glucose metabolism. Whereas co-overexpression of Slc38a1 and CLIP3 synergistically increased insulin-regulated glucose metabolism (Supplementary Fig. 3E and F). Collectively, these data suggest that Slc38a1 and CLIP3 do not function in the same signaling pathway.

A
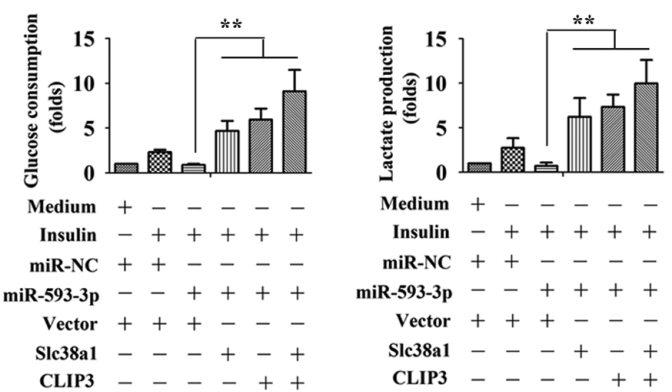

C

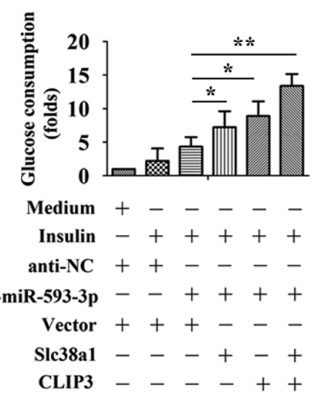

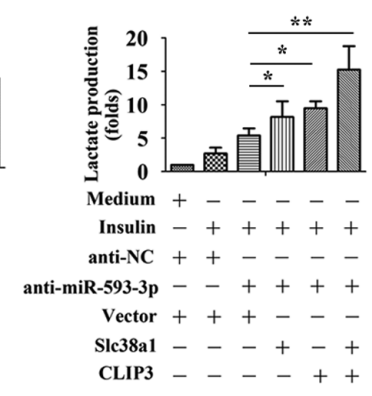

\section{Insulin regulates the expression of SIc38a1 and CLIP3 expression via miR-593-3p}

To verify whether insulin regulates miR-593-3p expression, HepG2 cells were exposed to 0, 100, 200 and $400 \mathrm{nM}$ of insulin for $6 \mathrm{~h}$, or exposed to $200 \mathrm{nM}$ of insulin for $0,3,6$ and $12 \mathrm{~h}$. Real-time PCR and Western blot results showed that insulin significantly inhibited miR-593-3p expression in a dose-dependent and time-dependent manner (Fig. 5A). Further experiments showed that insulin also induced Slc38a1 and CLIP3 expression in a dose-dependent and time-dependent manner (Fig. 5B and C). Whether the suppression of miR-593-3p by insulin could play a role in Slc38a1 expression was examined next. As shown in Fig. 5D, overexpression of miR-593-3p decreased the induction of Slc38a1 expression by insulin, and conversely anti-miR-593-3p increased insulininduced Slc38a1 expression. Similarly, miR-593-3p also controlled insulin-induced CLIP3 expression (Fig. 5E). Taken together, these data suggest that miR-593-3p is required for insulin-induced Slc38a 1 and CLIP3 expression.
B
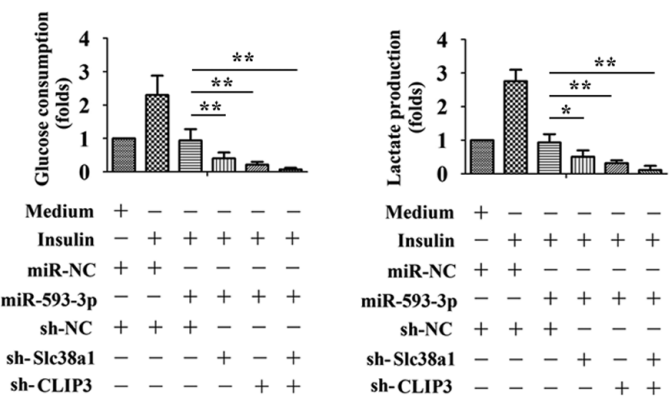

D

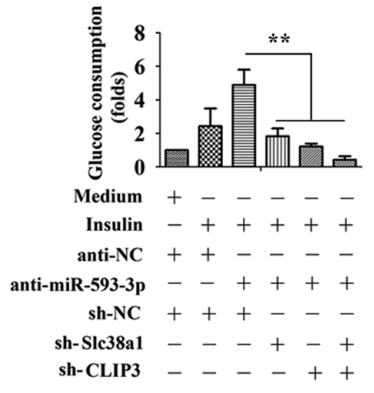

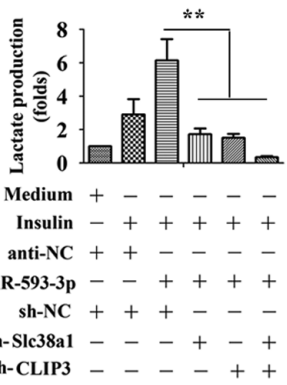

\section{Figure 6}

miR-593-3p regulated insulin-stimulated glucose consumption and lactate production by targeting SIc38a1 and CLIP3. (A) HepG2 cells were transfected with indicated miRNA and plasmid for $24 \mathrm{~h}$. Then, the cells were cultured in serum-free medium for $24 \mathrm{~h}$ and treated without or with insulin ( $200 \mathrm{nM})$ for $12 \mathrm{~h}$ before analysis of glucose consumption and lactate production levels. (B) Experiments were performed as in (A), except indicated shRNAs were used. ( $C$ and $D$ ) Experiments were performed as in (A) and (B), except indicated miRNA inhibitors were used. Bar graphs represent mean \pm s.D., $n=3$ $\left({ }^{* *} P<0.01 ; * P<0.05\right)$. Med, medium. 


\section{miR-593-3p inhibition of insulin-regulated glucose} energy metabolism is mediated by SIc38a1 and CLIP3

Because miR-593-3p, Slc38a1 and CLIP3 can regulate the insulin-stimulated glucose consumption and lactate production and both Slc38a1 and CLIP3 is a direct target gene of miR-593-3p, we next examined the role of miR-593-3p targeting of Slc38a1 and CLIP3 in the insulin-stimulated glucose consumption and lactate production. As shown in Fig. 6A, overexpression of Slc38a1 and CLIP3 abolished the inhibition of miR-593-3p mimic on insulin-regulated glucose metabolism. In contrast, the miR-593-3p mimic and the Slc38a1 and CLIP3 shRNAs synergistically suppressed insulin-stimulated glucose consumption and lactate production (Fig. 6B). The effect of the miR-593-3p, Slc38a1 and CLIP3 signaling pathways on the insulin-regulated glucose metabolism was further evaluated using the miR-593-3p inhibitor. As shown in Fig. 6C, Slc38a1 and CLIP3 overexpression significantly enhances the effect of the miR-593-3p inhibitor on the insulin-stimulated glucose consumption and lactate production. Conversely, low levels of glucose consumption and lactate production were observed in HepG 2 cells when Slc38a1 and CLIP3 was knocked down by shRNAs (Fig. 6D). The miR-593-3p decreases insulinregulated glucose energy metabolism through Slc38a1 and CLIP3 were not cell-type specific because similar results were observed in $\mathrm{C} 2 \mathrm{C} 12$ cells (Supplementary Fig. 4). These results suggest that the inhibition of Slc38a1 and CLIP3 expression is responsible for the ability of miR-593-3p to inhibit insulin-regulated glucose metabolism.

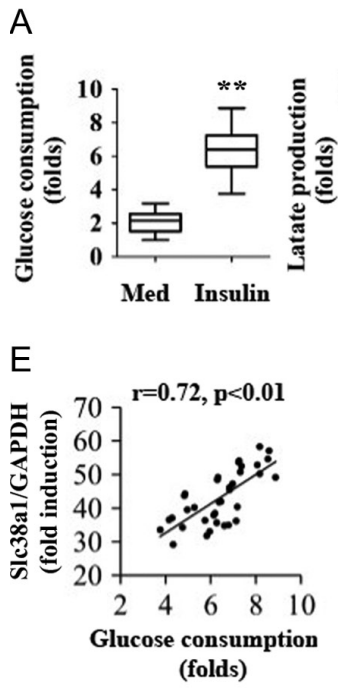

G



B

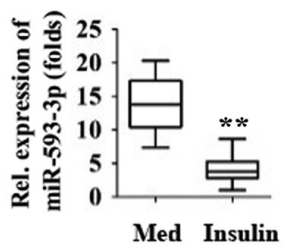

C

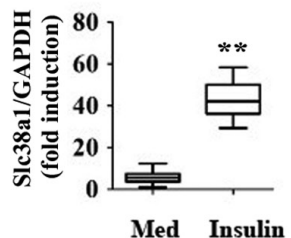

D

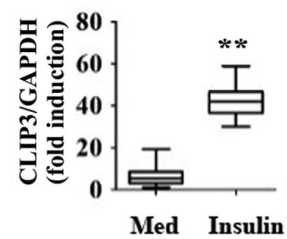

F
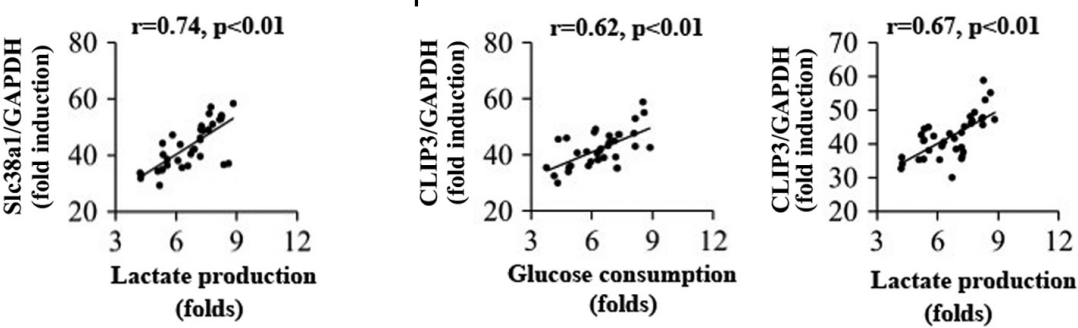

$\mathrm{H}$

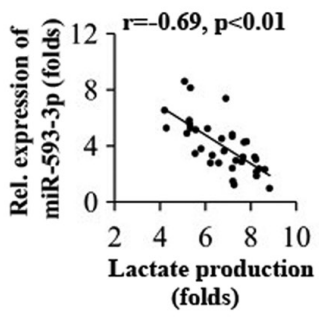



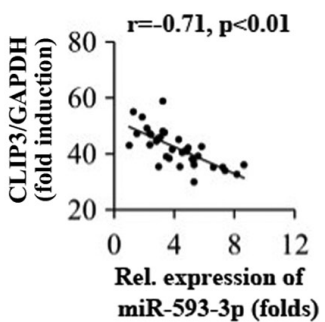

\section{Figure 7}

Analysis of the role of miR-593-3p, SIc38a1 and CLIP3 in insulin-stimulated glucose consumption and lactate production in human hepatocytes. (A) Human hepatocytes $(n=36)$ were cultured in serum-free medium for $24 \mathrm{~h}$ and treated without or with insulin (200 $\mathrm{nM}$ ) for $12 \mathrm{~h}$ before analysis of glucose consumption and lactate production levels. (B-D) Experiments were performed as in (A), except miR-593-3p levels (B), relative SIc38a1 (C) and CLIP3 (D) mRNA levels were determined by real-time PCR. (E-H) the relative SIc38a1 mRNA and glucose consumption or lactate production (E), the relative CLIP3 mRNA and glucose consumption or lactate production (F), the relative miR-593-3p levels and glucose consumption or lactate production (G), the relative miR-593-3p levels and Slc38a1 mRNA (left panel) or CLIP3 mRNA (right panel) (H), in insulin-treated human hepatocytes were subjected to Pearson's correlation analysis. Box plots illustrate medians with 25 and $75 \%$ and error bars for 5 and $95 \%$ percentiles. For A-H the lowest value was designated as 1 . Glucose consumption, lactate production, miR-593-3p, Slc38a1 and CLIP3 data are expressed as fold induction relative to the lowest value. Bar graphs represent mean \pm S.E.M., $n=3(* * P<0.01 ; * P<0.05)$.

http://jme.endocrinology-journals.org DOI: 10.1530/JME-16-0090
(C) 2016 Society for Endocrinology Printed in Great Britain
Published by Bioscientifica Ltd 


\section{Expression of miR-593-3p, SIc38a1 and CLIP3 in insulin- treated primary human hepatocytes}

To further elucidate their roles in insulin-regulated glucose metabolism, the expression levels of miR-593-3p, Slc38a1 and CLIP3 in insulin-treated primary human hepatocytes were determined. As shown in Fig. 7A, insulin significantly promoted glucose consumption and lactate production in primary human hepatocytes. Next, the effect of insulin on the expression of miR-593-3p, Slc38a1 and CLIP3 in primary human hepatocytes was explored. Real-time PCR results show that insulin inhibited miR-593-3p expression and induced Slc38a1 and CLIP3 expression of primary human hepatocytes (Fig. 7B, C and D). Interestingly, a statistically significant correlation was observed between Slc38a1 and CLIP3 levels and insulin-stimulated glucose consumption and lactate production (Fig. 7E and F). Conversely, low levels of miR-593-3p expression inversely correlated with high levels of Slc38a 1 and CLIP3 expression or insulin-stimulated glucose consumption and lactate production (Fig. 7G and H). miR-593-3p, Slc38a1 and CLIP3 expression was analyzed in 41 pairs of clinical liver cancer and 41 adjacent non-tumorous tissues. As determined by real-time PCR assay, miR-593-3p was downregulated and Slc38a1 and CLIP3 were upregulated in liver cancer tissues compared with non-tumorous tissues (Supplementary Fig. 5A, B and C). Elevated Slc38a1 and CLIP3 in the liver cancer tissues also closely correlated with low levels of miR-593-3p expression (Supplementary Fig. 5D and $\mathrm{E}$ ). This provides further evidence that alterations of miR-593-3p, Slc38a1 and CLIP3 expression are involved in the same pathway and could be involved in insulin-regulated glucose metabolism.

\section{Discussion}

Insulin has been reported to affect glucose metabolism by promoting glycolysis in some cell types (Saltiel \& Kahn 2001, Li et al. 2014). This has been found to be relevant in liver cancer (Saltiel \& Kahn 2001, Li et al. 2014). However, the mechanism remains to be elucidated. In this study, miR-593-3p was identified as a candidate miRNA that negatively regulates insulin-stimulated glucose metabolism. Further studies showed that miR-593-3p regulated insulin-stimulated glucose metabolism through direct targeting and reduction of the expression of Slc38a1 and CLIP3. To the best of the authors' knowledge, this is the first study to identify the role of the miR-593-3p/Slc38a1/CLIP3 axis in insulin-stimulated glucose metabolism.
Currently, three studies have reported functions of miR-593. Hu et al. found that miR-593 directly targets PROP1 mRNA in children with combined pituitary hormone deficiency (Hu et al. 2015). Fan et al. found that miR-593 is associated with mitochondrial fission and apoptosis in tongue squamous cell carcinoma cells (Fan et al. 2015). And, Ito et al. identified miR-593 as a candidate esophageal tumor-suppressive miRNA, via binding to the PLK1 3'-UTR (Ito et al. 2011). In this study, a new role of miR-593-3p was identified.

It was found that insulin promotes glucose consumption by regulation of miR-593-3p expression. Next, Slc38a1 and CLIP3 were identified as novel targets of miR-593-3p. Further studies demonstrated that both Slc38a1 and CLIP3 mediate insulin-regulated glucose metabolism. Correlations between miR-593-3p expression and insulin-regulated Slc38a1 and CLIP3 expression further support the conclusion that insulin upregulates Slc38a1 and CLIP3 expression through miR-593-3p expression. Slc38a1 is a glutamine transporter and was found to be related to cancer development. For example, Slc38a1 can induce the migration of human pancreatic cancer cells (Xie et al. 2013). Slc38a1 was found significantly elevated in human hepatocellular carcinoma and preneoplastic liver tissues (Kondoh et al. 2007). Slc38a1 is associated with lesser prognosis in patients with gastric cancer (Xie et al. 2014). However, as for CLIP3, little is known about the role of CLIP3 in cancer. This study found that Slc38a1 and CLIP3 were upregulated in liver cancer tissues compared with non-tumorous tissues (Supplementary Fig. 5). Insulin has been shown to induce glucose metabolism and is associated with a variety of cancer development in solid tumors (Matthews et al. 1985, Saltiel \& Kahn 2001). Because Slc38a1 and CLIP3 play an important role in insulin-regulated glucose metabolism and upregulated in liver cancer, the two genes may be the link between high insulin levels and elevated cancer risk. Moreover, our study may provide some useful information that Slc38a1 and CLIP3 may act as a potential strategy for therapeutic purpose in liver cancer treatment in the future.

Interestingly, we also examined the role of three known miR-593-3p target proteins, PROP1, MFF and PLK1, in insulin-stimulated glucose metabolism. None of these were involved in insulin-regulated glucose metabolism. In addition, we determined the relationship between Slc38a1 and CLIP3. Our data showed that Slc38a1 and CLIP3 are mutually independent proteins in insulin-regulated signaling. This suggests that miR-593-3p regulates the induction of glucose metabolism in liver cancer cells

Published by Bioscientifica Ltd 
through multiple mechanisms. Further studies are needed to address the molecular mechanism underlying the roles of Slc38a1 and CLIP3 in this process.

In summary, the results from this study suggest that insulin-promoted glucose metabolism through the miR-593-3p/Slc38a1/CLIP3 axis. The results describe a previously unknown mechanism for the regulation of insulin-promoted glucose metabolism, and also provide new insights on the role of miR-593-3p and its targeted genes in pathogenesis of liver cancer.

\section{Supplementary data}

This is linked to the online version of the paper at http://dx.doi.org/10.1530/ JME-16-0090.

\section{Declaration of interest}

The authors declare that there is no conflict of interest that could be perceived as prejudicing the impartiality of the research reported.

\section{Funding}

This work was supported by the Wuhan Science and Technology Plan (grant numbers: WSTP-2013062301010811).

\section{References}

Deng X, Wei H, Lou D, Sun B, Chen H, Zhang Y \& Wang Y 2012 Changes in CLIP3 expression after sciatic nerve injury in adult rats. Journal of Molecular Histology 43 669-679. (doi:10.1007/s10735-0129450-y)

El Ouaamari A, Baroukh N, Martens GA, Lebrun P, Pipeleers D \& van Obberghen E 2008 miR-375 targets 3'-phosphoinositide-dependent protein kinase-1 and regulates glucose-induced biological responses in pancreatic beta-cells. Diabetes 57 2708-2717. (doi:10.2337/db07-1614)

Fan S, Liu B, Sun L, Lv XB, Lin Z, Chen W, Chen W, Tang Q, Wang Y, Su Y, et al. 2015 Mitochondrial fission determines cisplatin sensitivity in tongue squamous cell carcinoma through the BRCA1miR-593-5p-MFF axis. Oncotarget 6 14885-14904. (doi:10.18632/ oncotarget.3659)

Hu Y, Wang Q, Wang Z, Wang F, Guo X \& Li G 2015 Circulating microRNA profiles and the identification of miR-593 and miR-511 which directly target the PROP1 gene in children with combined pituitary hormone deficiency. International Journal of Molecular Medicine 35 358-366. (doi:10.3892/ijmm.2014.2016)

Ito T, Sato F, Kan T, Cheng Y, David S, Agarwal R, Paun BC, Jin Z, Olaru AV, Hamilton JP, et al. 2011 Polo-like kinase 1 regulates cell proliferation and is targeted by miR-593* in esophageal cancer. International Journal of Cancer 129 2134-2146. (doi:10.1002/ ijc.25874)

Kondoh N, Imazeki N, Arai M, Hada A, Hatsuse K, Matsuo H, Matsubara O, Ohkura S \& Yamamoto M 2007 Activation of a system A amino acid transporter, ATA1/SLC38A1, in human hepatocellular carcinoma and preneoplastic liver tissues. International Journal of Oncology 31 81-87. (doi:10.3892/ijo.31.1.81)

Krol J, Loedige I \& Filipowicz W 2010 The widespread regulation of microRNA biogenesis, function and decay. Nature Reviews. Genetics 11 597-610. (doi:10.1038/nrg2843)
Lai EC 2002 Micro RNAs are complementary to 3' UTR sequence motifs that mediate negative post-transcriptional regulation. Nature Genetics 30 363-364. (doi:10.1038/ng865)

Lallemand-Breitenbach V, Quesnoit M, Braun V, El Marjou A, Pous C, Goud B \& Perez F 2004 CLIPR-59 is a lipid raft-associated protein containing a cytoskeleton-associated protein glycine-rich domain (CAP-Gly) that perturbs microtubule dynamics. Journal of Biological Chemistry 279 41168-41178. (doi:10.1074/jbc.M406482200)

Li W, Wang J, Chen QD, Qian X, Li Q, Yin Y, Shi ZM, Wang L, Lin J, Liu LZ, et al. 2013 Insulin promotes glucose consumption via regulation of miR-99a/mTOR/PKM2 pathway. PLoS ONE 8 e64924. (doi:10.1371/journal.pone.0064924)

Li Q, Liu X, Yin Y, Zheng JT, Jiang CF, Wang J, Shen H, Li CY, Wang M, Liu LZ, et al. 2014 Insulin regulates glucose consumption and lactate production through reactive oxygen species and pyruvate kinase M2. Oxidative Medicine and Cellular Longevity 2014504953. (doi:10.1155/2014/504953)

Liao L, Yang M, Qiu LL, Mou YR, Zhao JJ \& Dong JJ 2010 Appropriate insulin initiation dosage for insulin-naive type 2 diabetes outpatients receiving insulin monotherapy or in combination with metformin and/or pioglitazone. Chinese Medical Journal 123 3684-3688.

Lovis P, Roggli E, Laybutt DR, Gattesco S, Yang JY, Widmann C, Abderrahmani A \& Regazzi R 2008 Alterations in microRNA expression contribute to fatty acid-induced pancreatic beta-cell dysfunction. Diabetes 57 2728-2736. (doi:10.2337/db07-1252)

Matthews DR, Hosker JP, Rudenski AS, Naylor BA, Treacher DF \& Turner RC 1985 Homeostasis model assessment: insulin resistance and beta-cell function from fasting plasma glucose and insulin concentrations in man. Diabetologia 28 412-419. (doi:10.1007/ BF00280883)

McGivan JD \& Pastor-Anglada M 1994 Regulatory and molecular aspects of mammalian amino acid transport. Biochemical Journal 299 321-334. (doi:10.1042/bj2990321)

Moore CX \& Cooper GJ 1991 Co-secretion of amylin and insulin from cultured islet beta-cells: modulation by nutrient secretagogues, islet hormones and hypoglycemic agents. Biochemical and Biophysical Research Communications 179 1-9. (doi:10.1016/0006291X(91)91325-7)

Morita S, Horii T, Kimura M \& Hatada I 2013 MiR-184 regulates insulin secretion through repression of Slc25a22. PeerJ 1 e162. (doi:10.7717/ peerj.162)

Ogura M, Kakuda T, Takarada T, Nakamichi N, Fukumori R, Kim YH, Hinoi E \& Yoneda Y 2012 Promotion of both proliferation and neuronal differentiation in pluripotent P19 cells with stable overexpression of the glutamine transporter slc38a1. PLOS ONE 7 e48270. (doi:10.1371/journal.pone.0048270)

Saltiel AR \& Kahn CR 2001 Insulin signalling and the regulation of glucose and lipid metabolism. Nature 414 799-806. (doi:10.1038/414799a)

Sonoda N, Imamura T, Yoshizaki T, Babendure JL, Lu JC \& Olefsky JM 2008 Beta-Arrestin-1 mediates glucagon-like peptide-1 signaling to insulin secretion in cultured pancreatic beta cells. PNAS $\mathbf{1 0 5}$ 6614-6619. (doi:10.1073/pnas.0710402105)

Sorice M, Matarrese P, Manganelli V, Tinari A, Giammarioli AM, Mattei V, Misasi R, Garofalo T \& Malorni W 2010 Role of GD3CLIPR-59 association in lymphoblastoid $\mathrm{T}$ cell apoptosis triggered by CD95/Fas. PLoS ONE 5 e8567. (doi:10.1371/journal.pone.0008567)

Sugawara M, Nakanishi T, Fei YJ, Huang W, Ganapathy ME, Leibach FH \& Ganapathy V 2000 Cloning of an amino acid transporter with functional characteristics and tissue expression pattern identical to that of system A. Journal of Biological Chemistry 275 16473-16477. (doi:10.1074/jbc.C000205200)

Tavana O \& Zhu C 2011 Too many breaks (brakes): pancreatic beta-cell senescence leads to diabetes. Cell Cycle 10 2471-2484. (doi:10.4161/ cc.10.15.16741) http://jme.endocrinology-journals.org

DOI: 10.1530/JME-16-0090
๑ 2016 Society for Endocrinology Printed in Great Britain
Published by Bioscientifica Ltd 
Thirone AC, Huang C \& Klip A 2006 Tissue-specific roles of IRS proteins in insulin signaling and glucose transport. Trends in Endocrinology and Metabolism 17 72-78. (doi:10.1016/j. tem.2006.01.005)

Xie J, Chen Z, Liu L, Li P, Zhu X, Gao H \& Meng Z 2013 shRNAmediated Slc38a1 silencing inhibits migration, but not invasiveness of human pancreatic cancer cells. Chinese Journal of Cancer Research 25 514-519. (doi:10.3978/j.issn.10009604.2013.09.03)
Xie J, Li P, Gao HF, Qian JX, Yuan LY \& Wang JJ 2014 Overexpression of SLC38A1 is associated with poorer prognosis in Chinese patients with gastric cancer. BMC Gastroenterology 14 70. (doi:10.1186/1471230X-14-70)

Ying H, Kimmelman AC, Lyssiotis CA, Hua S, Chu GC, FletcherSananikone E, Locasale JW, Son J, Zhang H, Coloff JL, et al. 2012 Oncogenic Kras maintains pancreatic tumors through regulation of anabolic glucose metabolism. Cell 149 656-670. (doi:10.1016/j. cell.2012.01.058)

Received in final form 18 August 2016

Accepted 7 September 2016

Accepted Preprint published online 9 September 2016
Published by Bioscientifica Ltd. 